\title{
Optical and structural properties of Au-Ag islands films for plasmonic applications
}

\author{
J. Sancho-Parramon ${ }^{1 *}$, V. Janicki ${ }^{1}$, M. Lončarić ${ }^{1}$, H. Zorc ${ }^{1}$, P. Dubček ${ }^{1}$ and S. Bernstorff ${ }^{2}$ \\ ${ }^{1}$ Ruđer Bošković Institute, Bijenička 54, 10000 Zagreb, Croatia \\ ${ }^{2}$ Sincrotrone Trieste, SS 14 km 163.5, 34012 Basovizza (Trieste), Italy \\ *e-mail: jsancho@irb.hr
}

\begin{abstract}
Bimetallic islands films consisting of composite Au-Ag nanoparticles are deposited on glass substrates by electron beam evaporation. Broad tuning of the surface plasmon resonance (SPR) characteristics can be achieved by controlling film composition, deposition temperature and post-deposition thermal annealing. Optical and structural characterization of the samples enable to establish the link between the SPR and the morphological and compositional characteristics of nanoparticles.
\end{abstract}

\section{INTRODUCTION}

Gold and silver are the most widely used metals for nanoparticle-based plasmonic applications, such as chemical and biological sensing, luminescence enhancement or photovoltaic efficiency increase. Tuning of the SPR is usually achieved by controlling the size, shape and spatial arrangement of nanoparticles. Further tuning of the SPR over a wide energy range can be obtained using Au-Ag bimetallic nanoparticles, either as core-shell or as alloyed structure ${ }^{1}$. A simple way to obtain $\mathrm{Au}-\mathrm{Ag}$ nanoparticle systems is deposition of metals island films. These films can be considered as two-dimensional ensembles of metal clusters and are one of the most easy-to-prepare cases of nanostructured matter, as they can be obtained during the first stage of evaporation process. Recently, metal island films have been used as building blocks for photonic and plasmonic structures ${ }^{2}$. Typical growth of metal island films results into broad distribution of size and shape of particles that can be partially controlled by the deposition conditions and post-deposition treatments.

In the present work we study the optical properties of $\mathrm{Au}-\mathrm{Ag}$ metal island films deposited on glass substrates by electron beam evaporation. The influence of composition, deposition temperature and post-deposition annealing treatment is analyzed using spectrophotometry and spectroscopic ellipsometry for optical characterization, while the structural properties of the islands are determined using grazing-incidence small-angle $\mathrm{x}$-ray scattering (GISAXS). The results illustrate the strong correlation between optical and structural properties of the islands and evidence the possibility to widely tailor the SPR of these systems.

\section{EXPERIMENTAL}

Metal island films consisting of Au-Ag particles were deposited on 1-mm thick BK7 glass substrates by electron beam evaporation in a Varian 3117 deposition chamber. The Au-Ag films were deposited by the subsequent deposition of few nanometers of $\mathrm{Ag}$ and of Au. Due to the poor wetting of metals on glass substrate, the films follow an island-like growth mechanism (Volmer-Weber growth) rather than forming a compact film ${ }^{3}$. Films with different $\mathrm{Ag} / \mathrm{Au}$ composition were deposited with the following mass thickness (in nanometers): $2 / 6,4 / 4$ and 6/2. Films were deposited on substrates at room temperature $\left(\mathrm{T}_{\mathrm{d}}=25{ }^{\circ} \mathrm{C}\right)$ and pre-heated at $\mathrm{T}_{\mathrm{d}}=220^{\circ} \mathrm{C}$. 
After deposition, samples were annealed 20 minutes at $350^{\circ} \mathrm{C}$ in air in a Lindberg/Blue $\mathrm{M}$ furnace.

The surface plasmon resonance was characterized with photometric measurements (reflectance and transmittance at near-normal incidence) with a Perkin Elmer Lambda 25 spectrophometer in the range 300-1100 $\mathrm{nm}$. Ellipsometric measurements were performed at different angles of incidence $\left(45^{\circ}, 55^{\circ}, 65^{\circ}\right)$ in the range 300-2200 nm with a J. A. Woollam V-VASE spectroscopic ellipsometer. In addition, transmittance spectra at normal incidence were taken with the ellipsometer at the same sample point. Fitting of the ellipsometric and transmittance measurements allows determining the effective optical constants and thickness of the islands films. A multiple oscillator approach was used to represent the dispersion of the optical constants of the films. GISAXS measurements were carried out at the Austrian SAXS beam line at the Synchrotron ELETTRA in Trieste (Italy), using $8 \mathrm{keV}$ X-ray photon energy $(\lambda=0.154 \mathrm{~nm})$. The GISAXS intensity curves are obtained from the scattering pattern recorded by a two-dimensional charge-coupled device (CCD) sensitive detector. A motorized Al beam stop was positioned perpendicularly to the sample surface to reduce the transmitted and specularly reflected beams. The samples were mounted on a stepper-motor-controlled tilting stage with a step resolution of $0.001^{\circ}$ and measured at the chosen grazing angles.

\section{RESULTS AND DISCUSSION}

For all the samples, spectrophotometric measurements show strong optical extinction in the visible spectral range that can be associated to the surface plasmon resonance of islands (Figure 1). Additional absorption was observed in the UV spectral range, what can be related to the electronic interband transition of $\mathrm{Au}$ and $\mathrm{Ag}$. As deposited samples show a broad SPR that, in general, becomes narrower and blue-shifted upon thermal annealing (Table 1). This change is more evident for the islands films deposited at room temperature.
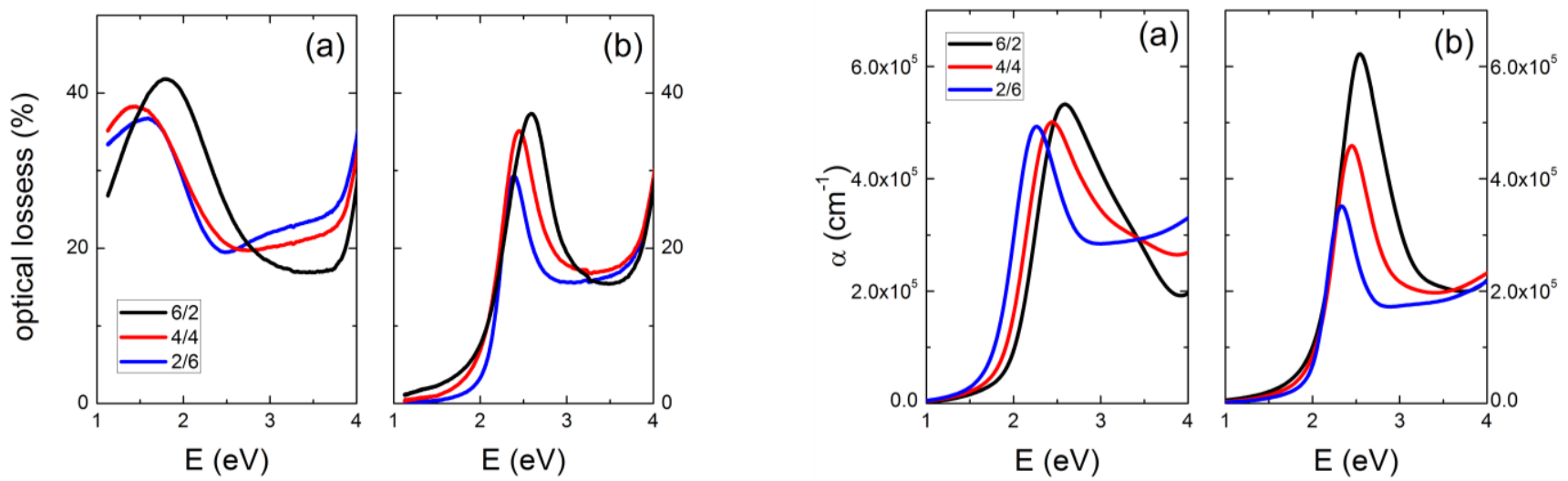

Figure 1. Optical losses of metal island films deposited at room temperature with $\mathrm{Ag} / \mathrm{Au}$ ratios 6/2 (black line) 4/4 (red line) and 2/6 (blue line): as deposited (a) and after thermal annealing (b).

Figure 2. Effective absorption coefficient obtained from ellipsometric measurements for the films deposited at $220{ }^{\circ} \mathrm{C}$ with $\mathrm{Ag} / \mathrm{Au}$ ratios 6/2 (black line) 4/4 (red line) and 2/6 (blue line): as deposited (a) and after thermal annealing (b).

The effective optical constants of the islands films extracted from spectroscopic ellipsometry agree with the results from spectrophotometry (Figure 2). In order to fit the ellipsometric data, two peaks are required to model the SPR for the as-deposited samples while only one peak is necessary for the annealed samples. This double-peak structure of the SPR for the as-deposited samples has been previously associated to a core-shell 
structure (one peak associated to the core material and the other to the shell material) ${ }^{4,5}$. Upon annealing, alloying of the particles takes place, leading to a single-peak $\mathrm{SPR}^{6,7}$. Each of these peaks was represented with two Gaussian oscillators in order to take into account inhomogeneous line broadening. In addition, one or two Gaussian oscillators were required to described the interband transitions, in connection with the interband transitions of pure $\mathrm{Au}$ (at 3 and $4 \mathrm{eV}$ ) and $\mathrm{Ag}(\sim 4.1 \mathrm{eV})^{8}$. The statistical uncertainty of the effective thickness as determined by ellipsometric measurements was between 1 and $5 \%$. At this point, it has to be remarked that the simultaneous use of ellipsometric and transmittance measurements permits reducing the otherwise strong correlation between optical constants and thickness for very thin absorbing films ${ }^{9}$.

GISAXS scattering patterns reveal that the film is composed of nanometric clusters distributed at the sample surface, as shown by the presence of two lobes of scattered light symmetric with respect to the incidence angle. For the annealed samples the intensity of the scattered light is more concentrated at smaller angles, suggesting that the particle size increases upon annealing. An estimation of the particle size can be done in terms of the so-called Guinier approximation, that gives the particle radius in the direction perpendicular $\left(R_{V}\right)$ and parallel $\left(R_{H}\right)$ to the sample surface from analysis of 1D intensity distributions in the two characteristic directions parallel and perpendicular to sample surface (Table 1$)^{10}$. In addition, the average interparticle distance $(D)$ can be estimated from the position of the maximum of scattered light ${ }^{10}$. As observed previously in metal island films ${ }^{11}$, samples deposited at room temperature show a more elliptical shape and smaller size than those deposited at hot substrates. Upon annealing, particle size and interparticle distance increases, indicating the particle growth. It is interesting to compare the results from the effective thickness found by ellipsometry and the value $2 R_{v}$ that could be associated to the thickness layer suggested by GISAXS. These two parameters are positively correlated but the value of the thickness found by ellipsometry is few nanometers larger than the one obtained by GISAXS. This discrepancy must be understood in terms of the different physical principles of both methods.
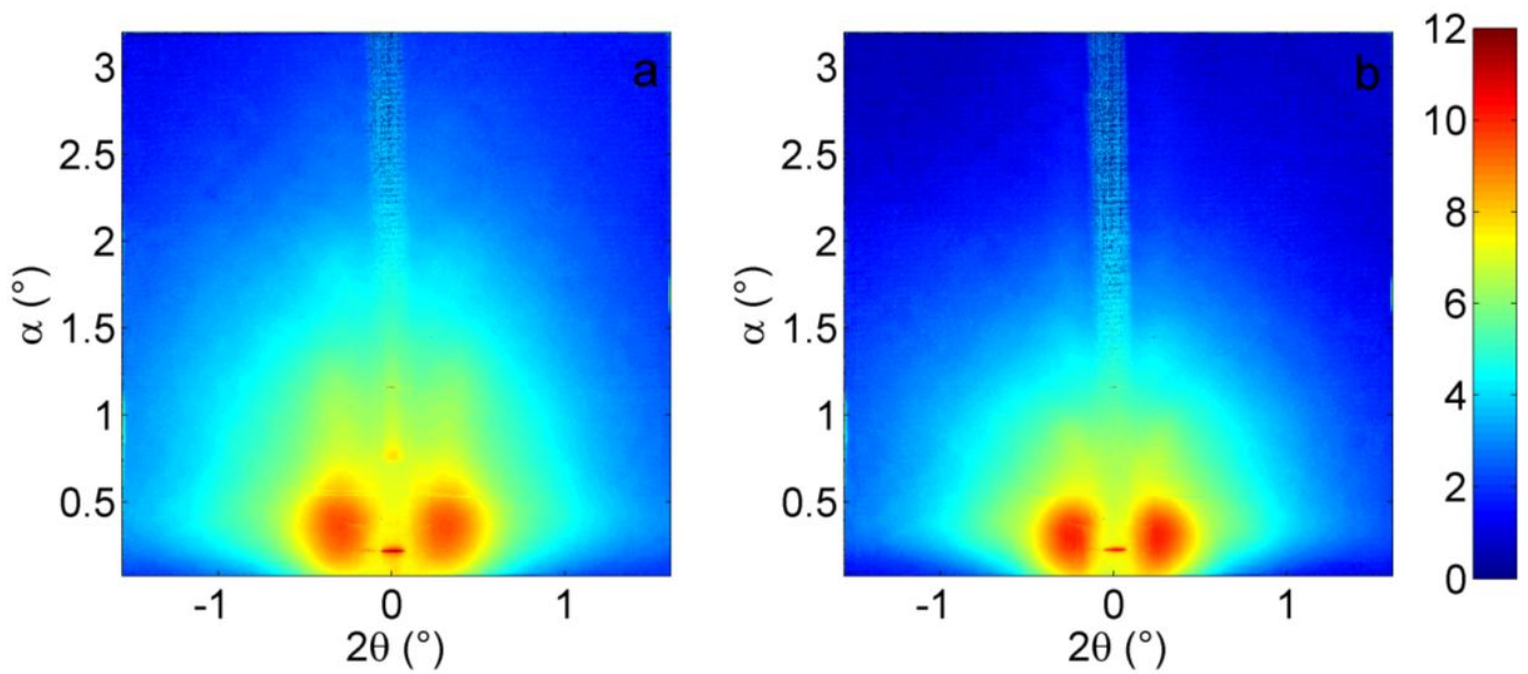

Figure 3: GISAXS intensity patterns (logarithmic scale) for the $\mathrm{Ag} / \mathrm{Au}$ film deposited at $220{ }^{\circ} \mathrm{C}$ with composition 2/6: as deposited (a) and after annealing (b). $\alpha$ and $2 \theta$ are the scattering angles in the direction perpendicular and parallel to the islands film, respectively.

The results show a strong interplay between the islands composition and shape and the observed optical properties. Thus, while the SPR of the films deposited at hot substrates is red-shifted with higher Au content, this trend is not observed for the films deposited at room temperature. In this case, the SPR peak appears to be more 
correlated with the degree of sphericity of the islands than with the Au content. Indeed, island films deposited at cold substrates have a small aspect ratio $\left(R_{V} / R_{H}\right)$ that strongly affects the $\mathrm{SPR}^{10}$.

Upon thermal annealing, SPR red-shift with higher Au content is observed for films deposited at both, room temperature and hot substrates. In this case, the SPR is well defined as a single and narrow peak that indicates the particle alloying. For single-material island films, increase of annealing induces increase of particle sphereicity and consequent blue-shift of the $\mathrm{SPR}^{10}$. In several of the samples studied here, the islands become more elliptical after annealing. This shape change can compensate the blue-shift due to alloying and even result in a red-shift of the SPR respect of the as-deposited films, as observed for the films with higher Ag content deposited at hot substrates. Since Ag islands grow more spherically than $\mathrm{Au}$, alloying of $\mathrm{Ag}-\mathrm{Au}$ islands may result in a more elliptical shape than the original core-shell particle, especially if the Ag core is close to spherical, as expected for the samples deposited at hot substrates.

\begin{tabular}{|c|c|c|c|c|c|c|c|}
\hline Td & Treatment & $\mathrm{Ag} / \mathrm{Au}$ & $\mathbf{R}_{\mathbf{V}}(\mathbf{n m})$ & $\mathbf{R}_{\mathbf{H}}(\mathbf{n m})$ & D (nm) & $\mathbf{t}(\mathbf{n m})$ & SPR max $(\mathrm{nm})$ \\
\hline \multirow[t]{6}{*}{$25^{\circ} \mathrm{C}$} & \multirow{3}{*}{$\begin{array}{c}\text { As } \\
\text { deposited }\end{array}$} & $2 / 6$ & 4.1 & 6.5 & 21.3 & 7.1 & 781 \\
\hline & & $4 / 4$ & 3.4 & 6.9 & 21.4 & 7.8 & 860 \\
\hline & & $6 / 2$ & 4.2 & 7.7 & 22.8 & 7.9 & 683 \\
\hline & \multirow[t]{3}{*}{ Annealed } & $2 / 6$ & 10.5 & 17.5 & 53.3 & 16.1 & 519 \\
\hline & & $4 / 4$ & 11.6 & 15.2 & 45.6 & 14.2 & 506 \\
\hline & & $6 / 2$ & 9.1 & 15.5 & 45.3 & 11.5 & 477 \\
\hline \multirow[t]{6}{*}{$220^{\circ} \mathrm{C}$} & \multirow{3}{*}{$\begin{array}{c}\text { As } \\
\text { deposited }\end{array}$} & $2 / 6$ & 6.9 & 10.9 & 29.1 & 10.4 & 568 \\
\hline & & $4 / 4$ & 8.3 & 11.8 & 29.7 & 11.6 & 522 \\
\hline & & $6 / 2$ & 9.3 & 10.7 & 29.1 & 13.1 & 494 \\
\hline & \multirow[t]{3}{*}{ Annealed } & $2 / 6$ & 9.9 & 13.3 & 36.6 & 13.1 & 537 \\
\hline & & $4 / 4$ & 10.2 & 15.1 & 41.3 & 12.0 & 523 \\
\hline & & $6 / 2$ & 9.4 & 14.5 & 39.1 & 11.8 & 504 \\
\hline
\end{tabular}

Table 1: Summary of results from GISAXS analysis (vertical radius $R_{V}$, horizontal radius $R_{H}$ and interparticle distance $D$ ) ellipsometry (thickness $t$ ) and photometric measurements (SPR wavelength) for the studied samples.

\section{CONCLUSIONS}

The optical and structural properties of bimetallic $\mathrm{Ag} / \mathrm{Au}$ island films obtained by electron beam evaporation have been determined. A strong interplay between the SPR properties and the islands shape and composition is observed. As required for many plasmonic applications, the SPR of these systems can be widely tuned depending on the deposition conditions and post-deposition annealing treatments.

\section{REFERENCES}

1. F. Hubenthal, N. Borg and F. Träger "Optical properties and ultrafast electron dynamics in gold-silver alloy and core-shell nanoparticles”, Appl. Phys. B, Vol. 93, 39-45, 2008.

2. V. Janicki, J. Sancho-Parramon, F. Peiró and J. Arbiol "Three-dimensional photonic microstructures produced by electric field assisted dissolution of metal nanoclusters in multilayer stacks", Appl. Phys. B, Vol. 98, 93-98, 2010.

3. N. Kaiser "Review of the fundamentals of thin film growth" App. Optics, Vol. 41, No. 16, 3053-3060, 2002.

4. M. Moskovits, I. Srnova-Sloufova and B. Vlckova "Bimetallic Ag-Au nanoparticles: Extracting meaningful 
opticall constants from the surface-plasmon extinction spectrum" J. Chem. Phys. Vol. 116, No. 23, 10435-10446, 2002.

5. W. Benten, N. Nilius, N. Ernst and H. -J. Freund "Photon emission spectroscopy of single oxide-supported Ag-Au alloy clusters" Phys. Rev. B, Vol. 72, 045403, 2005.

6. K. Baba, T. Okuno and M. Miyagi "Silver-gold compound metal island films prepared by using a two-step evaporation method" Appl. Phys. Lett., Vol. 62, No. 5, 437-439, 1992.

7. K. Baba, Y. Ohkuma, T. Yonezawa and M. Miyagi "Silver-based compound metal island films for write-once optical data-storage media" Appl. Optics, Vol. 40, No. 16, 2796-2804, 2001.

8. P. B. Johnson and R. W. Christy "Optical constants of the noble metals" Phys. Rev. B, Vol. 6, 4370, 1972.

9. J. N. Hilfiker, N. Singh, T. Tiwald, D. Convey, S. M. Smith, J. H. Baker, and H. G. Tompkins "Survey of methods to characterize thin absorbing films with spectroscopic ellipsometry" Thin Solid Films, Vol 516, 7979, 2008.

10. M. Lončaric, et. al. "Optical and structural characterization of silver islands films on glass substrates", Vacuum, Vol. 84, 188-192, 2009.

11. R. R. Singer, A. Leitner, F. R. Aussenegg "Structure analysis and models for optical constants of discontinuous metallic silver films" J. Opt. Soc. Am. B Vol. 12, No. 2, 220-228, 1995. 